\title{
Exact Energy Bands and Fermi Surfaces of Separable Abelian Potentials
}

E D Belokolos, ${ }^{*}$ J C Eilbeck,$^{\dagger}$ V.Z. Enolskii, ${ }^{\ddagger}$ and M Salerno ${ }^{\S}$

\begin{abstract}
We present a general theory for multidimensional Schrödinger equations with separable Abelian potentials with an arbitrary number of gaps in the spectrum. In particular we derive general equations which allow to express the energy and the wave vectors in the BZ as a function of the spectral parameters. By using the solutions of these equations, we show how to construct the energy bands and the Fermi surfaces in the first Brillouin zone of the reciprocal lattice. As illustrative examples we consider the case of $2 \mathrm{D}$ separable potentials with 1-, 2-, and 3-gaps in the spectrum. The method can be applied to crystals with a cubic or a rectangular parallelogram Wigner-Seitz cell in arbitrary dimensions. The possibility to generalize the theory to other crystal symmetries is also briefly discussed.
\end{abstract}

*Department of Theoretical Physics, NASU Institute of Magnetism 36-b Vernadsky str., Kiev-680, 03142,Ukraine. email: bel@imag.kiev.ua

${ }^{\dagger}$ Department of Mathematics, Heriot-Watt University, Riccarton, Edinburgh EH14 4AS, UK. email:J.C.Eilbeck@hw.ac.uk

${ }^{\ddagger}$ Department of Theoretical Physics, NASU Institute of Magnetism 36-b Vernadsky str., Kiev-680, 03142,Ukraine. email: vze@imag.kiev.ua

§Dipartimento di Scienze Fisiche "E.R.Caianiello" and INFM Unita' di Salerno, via S. Allende, I-84081, Baronissi (SA), Italy. email: salerno@sa.infn.it 
PACS: 02.10.Rn, 02.30.Jr, 71.18+y, 71.20-b 


\section{INTRODUCTION}

The general theory of the many-dimensional Schrödinger operator was established many years ago, i.e. immediately after the advent of quantum mechanics. Since the pioneering work of F. Bloch [1], in which the spectral properties of these operators were first recognised to be of fundamental importance for the quantum theory of solids, much work has been done. This lead to the development of the band theory, one of the pillars of modern Solid State Physics. In spite of this, only a few examples are known of Schrödinger operators with periodic potentials, allowing exact analytical calculations of energy bands and Fermi surfaces.

Although numerical and self-consistent methods to compute the band structure of a solid with good accuracy exist, it is desirable to have exact solutions of the Schrödinger equation. These solutions, for some physically relevant multidimensional periodic potentials, are useful not only for comparison with approximate approaches, but also for further developments of the general theory. This would allow, for example, to get a deep understanding of the spectral manifolds and of the corresponding eigenfunctions, as well as of their geometrical and analytical properties.

From a mathematical point of view, the most interesting periodic potentials are the ones which can be expressed in terms of Abelian functions (Abelian potentials). Abelian functions are multiply periodic functions being defined on complex tori, which can be considered in the context of the problem as a siutable complexification of the real crystal lattice and its dual. In some sense they are the analogues of the potentials in classical mechanics which allow a complexification of the Liouville tori leading to Jacobians and Abelian functions and hence to algebraic integrable systems. It is remarkable that although the theory of such potentials was developed long before the ad- 
vent of quantum mechanics, they have been almost ignored by the Solid State Physics community. On the contrary, we think these potentials are important and should be considered in Solid State Physics as being the equivalent of Kepler's problem for atomic physics. They give the opportunity to study many questions related to the electronic properties of solids in a clear geometrical and analytical form.

In this paper we are interested in multidimensional separable generalizations of the simplest possible Abelian potential, i.e. the one expressed as Weierstrass elliptic function $\wp(z)$, with one real and one pure imaginary period. The Schrödinger operator with such a potential has exactly 1-gap spectrum and allows to obtain eigenfunctions in an explicit form (in the mathematical literature this equation is known as the 1D Lamé equation). This spectral problem is essentially easier and more clear than e.g. the well known Kronig-Penney problem, and can be used as a building block of a multidimensional theory. Our extensions to this problem are twofold: one is to consider multi-gap spectrum (a well studied problem), and the second to extend these multi-gap results to higher dimensions.

The investigation of the 1D Lamé equation was very well developed in the classical monographs of Halphen [2], Krause [3], Forsyth [4], and Whittaker and Watson [5]. Arscott [6,7] has surveyed results on the Jacobian form of the Lamé equation. The Lamé equation has also figured prominently in a number of recent publications devoted to study of completely integrable systems of KdV type: Krichever [8], Eilbeck and Enol'skii [9], Enol'skii and Kostov [10], Smirnov [11], Gesztesy and Weikard [12,13], Zabrodin [14], and others.

The aim of the present paper is to present the general theory for multidimensional Schrödinger equations with separable Abelian potentials with an arbitrary number of gaps in the spectrum (by separable here we mean those potentials which can be written as a sum of $N$ 1-dimensional finite-gap poten- 
tial, one for each spatial dimension). The introduction of separable Abelian potentials and their application to problems of solid state physics was also considered in $[15,16]$ for potentials with one and two gaps in the spectrum. Our results allow us to express the energy and the wave vectors in the Brillouin Zone (BZ) as a function of the spectral parameters. The edges of the bands also follow from these equations as roots of specific polynomials. From a mathematical point of view this corresponds to the problem of constructing a hyperelliptic $N$-sheeted covering, $N=g(g+1) / 2$ over a torus (elliptic curve), first considered by Jacobi and developed further by Weierstrass, Poincare and others, see [17] and [18] for a detailed exposition.

By using the solutions of these equations for the spectral parameters, we show how to construct the energy bands and the Fermi surfaces in the first Brillouin zone of the reciprocal lattice. As illustrative examples we consider the case of $2 \mathrm{D}$ separable potentials with a 1-, 2-, and 3-gap spectrum. The method, although not restricted to $2 \mathrm{D}$, can be applied only to crystals with a cubic or a rectangular parallelogram Wigner-Seitz cell. Possible physical applications of our theory, as well as its generalization to other crystal symmetries will be briefly discussed at the end of the paper.

The paper is organised as follow. In Section 2 we present the mathematical theory of separable N-dimensional Abelian potentials and the general equations valid in the case of arbitrary gaps in the spectrum. In Section 3 we present detailed calculations of bands and Fermi curves for 2D lattices with both square and rectangular symmetry. In the Conclusion we discuss possible physical applications of these results to different problems.

\section{SEPARABLE ABELIAN G-GAP PERIODIC POTENTIALS}

The $N$-dimensional Schrödinger operator with the separable potential 


$$
U(x)=\sum_{k=1}^{N} U_{k}\left(x_{k}\right), \quad x=\left(x_{1}, \ldots, x_{N}\right)
$$

has the form

$$
\left(-\Delta+\sum_{k=1}^{N} U_{k}\left(x_{k}\right)\right) \Psi(x)=E \Psi(x), \quad x=\left(x_{1}, \ldots, x_{N}\right),
$$

and therefore has the eigenfunctions of the form:

$$
\Psi\left(x_{1}, \ldots, x_{n}\right)=\prod_{k=1}^{N} \psi_{k}\left(x_{k}\right),
$$

where the $\psi\left(x_{k}\right)$ satisfy the ordinary differential equations

$$
-\frac{d^{2} \psi_{k}}{d x_{k}^{2}}+U_{k}\left(x_{k}\right) \psi_{k}\left(x_{k}\right)=E_{k} \psi_{k}\left(x_{k}\right), \quad k=1, \ldots, N .
$$

The energy $E$ is of the form

$$
E=\sum_{i=1}^{N} E_{i}=\sum_{i=1}^{N} E\left(k_{i}\right),
$$

where $k=\left(k_{i}, \ldots, k_{N}\right)$ is the wave vector. In the limiting case, when $E\left(k_{i}\right)=$ $k_{i}^{2}$ and therefore

$$
E=\sum_{i=1}^{N} k_{i}^{2}
$$

our approach corresponds to the well known Harrison pseudopotential approach in a theory of electron spectral properties of metals [19]. Hence in this sense our method is a straightforward generalization of the Harrison method.

For simplicity we first discuss the properties of the 1-dimensional potentials, the construction blocks for the separable N-dimensional potentials in Eq. (1), by considering the 1-gap Lamé potential $U(x)=2 \wp(x)$

$$
-\frac{d^{2} \Psi(x)}{d x_{k}^{2}}+2 \wp(x) \Psi(x)=E \Psi(x)
$$

where $\wp(x)$ is the Weierstrass elliptic function characterized by the curve $w^{2}=4 t^{3}-g_{2} t-g_{3} \equiv 4\left(t-e_{1}\right)\left(t-e_{2}\right)\left(t-e_{3}\right)$ with parameters $g_{2}, g_{3}$ given by

$$
g_{2}=-4\left(e_{1} e_{2}+e_{1} e_{3}+e_{2} e_{3}\right), \quad g_{3}=4 e_{1} e_{2} e_{3}, \quad e_{1}+e_{2}+e_{3}=0
$$


(below we will show how these parameters are related to the boundaries of the spectra). It is known that the eigenfunction and the corresponding eigenvalues of this equation can be expressed as [5]

$$
\Psi(x ; a)=\frac{\sigma(a-x)}{\sigma(x) \sigma(a)} \exp \{x \zeta(a)\}, \quad E=\wp(a),
$$

where $a$ denotes a spectral parameter and $\sigma$ the Weierstrass $\sigma$-function related to the Weierestrass $\wp$ and $\zeta$ functions by the relations

$$
\zeta(u)=\frac{\partial}{\partial u} \ln \sigma(u), \quad \wp(u)=-\frac{\partial^{2}}{\partial u^{2}} \ln \sigma(u)=-\frac{\partial}{\partial u} \zeta(u) .
$$

The above wave functions can be rewritten in the form of a Bloch function as

$$
\Psi(i x ; a)=e^{i k(a) x} B(i x ; a), \quad B(i x ; a)=\frac{\sigma(a-i x)}{\sigma(i x) \sigma(a)} \exp \left\{i \frac{\eta^{\prime}}{\omega^{\prime}} a x\right\}
$$

where we take $x \rightarrow i x$ and introduced the wave vector $k(a)$ as

$$
k(a)=\zeta(a)-\frac{\eta^{\prime}}{\omega^{\prime}} a
$$

Note that $x$ imaginary does not effect the reality of the potential and it implies that the periodicity of the potential is $\operatorname{Im}\left(2 \omega^{\prime}\right)$ where $2 \omega^{\prime}$ denotes the imaginary period of the $\wp$ function. The ratio $\eta^{\prime} / \omega^{\prime}$ is real since $\eta^{\prime}$, the second imaginary period, is purely imaginary. One can then easily prove that (9) is nothing but the Floquet theorem [20] for the 1-gap Lamé potential. Indeed, by using the periodicity property of the $\sigma$ function

$$
\sigma\left(u+2 m \omega+2 m^{\prime} \omega^{\prime}\right)=(-1)^{m m^{\prime}+m+m^{\prime}} \sigma(u) e^{\left(u+m \omega+m^{\prime} \omega^{\prime}\right)\left(2 \eta m+2 \eta^{\prime} m^{\prime}\right)}
$$

and denoting the translation operator by one period of the potential by $T_{\operatorname{Im}\left(2 \omega^{\prime}\right)}$, we have that

$$
T_{\operatorname{Im}\left(2 \omega^{\prime}\right)} \Psi(i x ; a)=\Psi\left(i x+2 \omega^{\prime} ; a\right)=e^{i k(a) \operatorname{Im}\left(2 \omega^{\prime}\right)} \Psi(i x ; a)
$$

from which we see that $\Psi$ is an eigenfunction of the translation operator associated with the eigenvalue $e^{i k(a) \operatorname{Im}\left(2 \omega^{\prime}\right)}$ (this implies that $B(i x ; a)$ is a 
periodic function with period $\left.\operatorname{Im}\left(2 \omega^{\prime}\right)\right)$. Note that for the multidimensional separable wavefunction (3), eq. (12) automatically leads to the usual Bloch theorem [1]. An equation for the spectral parameter $a$ can be obtained by observing that the function

$$
\Lambda(u)=\wp(u)-\wp(a)
$$

is a solution of the equation

$$
\frac{d^{3} \Lambda}{d^{3} u}-4(2 \wp(u)+E) \frac{d \Lambda}{d u}-4 \wp^{\prime}(u) \Lambda=0
$$

Inserting (13) into (14) gives the expression for the energy $E$ in terms of the quantity $a$

$$
E=\wp(a)
$$

In this case we simply obtain the relation between the energy and the spectral parameter. However, in the general $g$-gap case, we will get a set of $g$ equations among the spectral parameters. For the $g=1$ case, Eq. (15) uniquely determines the energy bands. The reality condition of the energy and the properties of the $\wp$ function imply that the spectrum must lie in the intervals $\left[e_{3}, e_{2}\right]$ and $\left[e_{1}, \infty\right]$ (see below). Note that the equations (10) and (15) express the energy $E$ in terms of the wave vector $k$ in parametric form.

These expressions can be easily generalized to the case of the one dimensional Schrödinger equation with $g$-gap Lamé potentials $U(x)=g(g+1) \wp(x)$

$$
-\frac{d^{2} \Psi\left(x_{k}\right)}{d x_{k}^{2}}+g(g+1) \wp\left(x_{k}\right) \Psi\left(x_{k}\right)=E \Psi\left(x_{k}\right) .
$$

One can check that the eigenfunctions can be written as

$$
\Psi\left(x, a_{1}, \ldots, a_{g}\right)=e^{i k\left(a_{1}, \ldots, a_{g}\right) x} \prod_{j=1}^{g} \frac{\sigma\left(a_{j}-i x\right)}{\sigma(i x) \sigma\left(a_{j}\right)} \exp \left\{i x \sum_{j=1}^{g} \frac{\eta^{\prime}}{\omega^{\prime}} a_{j}\right\}
$$

with the wavevector $k$ given by the expression 


$$
k\left(a_{1}, \ldots, a_{g}\right)=\sum_{j=1}^{g}\left[\zeta\left(a_{j}\right)-\frac{\eta^{\prime}}{\omega^{\prime}} a_{j}\right] .
$$

The equations for the spectral parameters $a_{j}, j=1, \ldots, g$ can be determined from the fact that

$$
\Lambda(u)=\prod_{j=1}^{g}\left[\wp(u)-\wp\left(a_{j}\right)\right]
$$

is a solution of the equation

$$
\frac{d^{3} \Lambda}{d^{3} u}-4(g(g+1) \wp(u)+E) \frac{d \Lambda}{d u}-2 g(g+1) \wp^{\prime}(u) \Lambda=0 .
$$

Inserting (18) into (19) gives the following system of algebraic equations for the quantities $\wp\left(a_{j}\right)$

$$
\alpha_{k} E S_{k-1}+\beta_{k} g_{2} S_{k-2}+\gamma_{k} g_{3} S_{k-3}-\delta_{k} S_{k}=0, \quad k=1,2, \ldots, g,
$$

where $S_{k}$ denotes the $k$-th elementary symmetric function

$$
S_{k}=\sum_{j_{1}<j_{2}<\ldots<j_{k}}^{g} \wp\left(a_{j_{1}}\right) \wp\left(a_{j_{2}}\right), \ldots \wp\left(a_{j_{k}}\right),
$$

and

$$
\begin{aligned}
\alpha_{k} & =4(-1)^{k-1}(k-g-1), \\
\beta_{k} & =\frac{1}{2}(-1)^{k-1}\left(2(g-(k-4))^{3}-15(g-(k-4))^{2}+37(g-k+4)-30\right), \\
\gamma_{k} & =(-1)^{k}(g-k+3)(g-k+2)(g-k+1), \\
\delta_{k} & =2 k(-1)^{k}\left(4 g^{2}+2 g(2-3 k)+2 k^{2}-3 k+1\right) .
\end{aligned}
$$

(Note that in Eq. 20 we assume that $S_{-k}=0$ and $S_{0}=1$ ). One can easily check that the first equation (i.e. $k=1$ ) of the system in (20) gives the energy $E$ in terms of the quantities $a_{j}, j=1, \ldots, g$

$$
E=(2 g-1) \sum_{j=1}^{g} \wp\left(a_{j}\right),
$$

while the second and third equations $(\mathrm{k}=2,3)$ express the invariants $g_{2}, g_{3}$, in terms of the energy and the quantities $a_{j}, j=1, \ldots, g$, 


$$
\begin{gathered}
g_{2}=\frac{8}{g}\left\{\sum_{j=1}^{g} \wp^{2}\left(a_{j}\right)+\frac{S_{2}}{g-1}\right\}, \\
g_{3}=\frac{8}{g}\left\{\frac{2 g-3}{2 g} \sum_{i=1}^{g} \wp^{3}\left(a_{i}\right)-\frac{1}{g-1} \sum_{i<j}^{g}\left(\wp^{2}\left(a_{i}\right) \wp\left(a_{j}\right)+\wp\left(a_{i}\right) \wp^{2}\left(a_{j}\right)\right)-\frac{3(2 g-3)}{(g-1)(g-2)} S_{3}\right\}
\end{gathered}
$$

The other $g-3$ equations establish additional relations between the $a_{j}$. To construct the energy bands as function of $k$ one must solve the above system of algebraic equations for the $\wp\left(a_{j}\right)^{\prime} s$ together with a condition for the reality of the energy. In the procedure one must invert the $\wp$ function to get the $a_{j}$, and use equations to relate the energy $E$ to the wave vectors $k$ in a parametric form. Although this looks complicated it can be easily performed on a computer using one of the many parametric plot packages available.

Before presenting explicit numerical results let us discuss how to derive from Eq. (20) the expression for the edges of the bands. We wish to write these bands as a function of the parameters $e_{1}, e_{2}, e_{3}$ characterising the underlying elliptic curve. In order to do this one must add to the above general formulas an extra condition which guarantee the reality of the energy (note that since the energy is unknown, we actually have $g$ equations for $g+1$ unknowns). This can be done by fixing one of the spectral parameters $a_{i}$, say $a_{1}$, so that $\wp\left(a_{1}\right)=e_{i}$. One can easily show that this choice will guarantee the reality of the energy. By constructing the Groebner basis for the polynomial in Eqs (20) one can eliminate the $g-1$ variables $\wp\left(a_{i}\right), i=2, \ldots, g$, in favour of the energy. This will lead to factorized polynomials which for $g=1,2,3$, are given respectively by

$$
\begin{aligned}
& 4 \prod_{i=1}^{3}\left(E-e_{i}\right), \\
& \left(E^{2}-3 g_{2}\right) \prod_{i=1}^{3}\left(E+3 e_{i}\right), \\
& E \prod_{i=1}^{3}\left(E^{2}-6 e_{i} E+45 e_{i}^{2}-15 g_{2}\right) .
\end{aligned}
$$


It is possible to set up a general procedure to determine these polynomials (and therefore the edges of the bands) for potentials with an arbitrary number of gaps. Thus, for example, for $g=5,7,9$ we obtain

$$
\begin{gathered}
\left(E^{2}-27 g_{2}\right) \prod_{i=1}^{3}\left[E^{3}-15 e_{i} E^{2}+\left(315 e_{i}^{2}-132 g_{2}\right) E+540 g_{3}+675 e_{i}^{3}\right], \\
\left(E^{3}-196 g_{2} E+2288 g_{3}\right) \prod_{i=1}^{3}\left[E^{4}-28 e_{i} E^{3}+\left(1134 e_{i}^{2}-574 g_{2}\right) E^{2}+\right. \\
\left.\left(34100 e_{i}^{3}-5116 e_{i} g_{2}\right) E+59150 e_{i}^{2} g_{2}-528983 e_{i}^{4}+22113 g_{2}{ }^{2}+88452 g_{3} e_{i}\right] \\
\left(E^{4}-774 g_{2} E^{2}+21600 g_{3} E+41769 g_{2}^{2}\right) \prod_{i=1}^{3}\left[E^{5}-45 e_{i} E^{4}+\left(2970 e_{i}^{2}-1764 g_{2}\right) E^{3}+\right. \\
\left(25380 e_{i} g_{2}-202230 e_{i}^{3}\right) E^{2}-\left(9461259 e_{i}^{4}-402624 g_{2}^{2}-1610496 g_{3} e_{i}-1210356 e_{i}^{2} g_{2}\right) E \\
\left.-20241900 e_{i}^{3} g_{2}+37013760 e_{i}^{2} g_{3}-76485465 e_{i}^{5}+9253440 e_{i} g_{2}^{2}\right]
\end{gathered}
$$

Notice that the problem of constructing these polynomials is related to the problem of constructing coverings for hyperelliptic curves on a $g$-sheetedly torus considered by Jacobi, Weierstrass and Poincaré. For Lamé potentials with an arbitrary number of gaps, a solution of this problem can be obtained from the above equations as will be discussed in a separate paper. Note that the system of Eqs. in (20) refers to the $g$-gap 1D potential i.e. to the building blocks of the higher dimensional cases. In the higher dimensional separable case one must, of course, consider several of such systems, with different spectral parameters and different elliptic curves, one for each dimension. In the case in which the curves coincide, the systems of equations and their corresponding solutions will obviously coincide. These will be discussed in the next section. 


\section{ENERGY BANDS AND FERMI CURVES FOR 2D SEPARABLE POTENTIALS}

From a physical point of view, one is interested in computing the shapes of the energy bands and the corresponding Fermi surfaces as a function of the wave numbers in the first BZ. From this knowledge, important physical properties of the system will follow, such as the density of states, the electron effective mass, transport properties, etc. In this section we will show how this can be done exactly for $2 \mathrm{D}$ separable elliptic potentials with one, two and three gaps in the spectrum, using the theory described above (for simplicity we restrict ourselves to the $2 \mathrm{D}$ case, but the construction is quite general and can be applied to 3D as well). This will cover the cases of crystals with a square or rectangular Wigner-Seitz cell and with 2, 3, 4 energy bands, respectively.

More specifically, let us consider the potential

$$
U(x, y)=g(g+1)(\wp(i x+\omega)+\wp(i y+\tilde{\omega}))
$$

associated with two elliptic curves $w^{2}=4 t^{3}-g_{2} t-g_{3}$ and $\tilde{w}^{2}=4 t^{3}-\tilde{g}_{2} t-\tilde{g}_{3}$ (one for each spatial direction) with $g_{2}, g_{3}$ (and similarly for $\tilde{g}_{2}, \tilde{g}_{3}$ and $\tilde{e}_{i}, i=$ $1,2,3)$ related to the gap edges $e_{i}, i=1,2,3$ by Eqs. (7). Note that in order to have a real and nonsingular potential for all $x, y \in R$, we have taken the argument of the $\wp$ functions appearing in the potential, along the imaginary axis shifted by the half real periods $\omega, \tilde{\omega}$ of the corresponding elliptic curve (recall that the $\wp$ function is singular at the origin). This implies that the periodicity in the $x$ and $y$ directions are just the two imaginary periods $2 \omega^{\prime}, 2 \tilde{\omega}^{\prime}$.

We shall first consider the case of identical elliptic curves, this corresponding to lattices with square Wigner-Seitz cells (the periodicity of the potential 
in the $x$ and $y$ direction being the same). The case of different curves, associated with rectangular Wigner-Seitz cells, will be discussed at the end of this section. In the following calculation we fix the parameters $e_{1}=2, e_{2}=-0.5$, $e_{3}=-1.5$ (and $\tilde{e}_{i}=e_{i}$ ), this correspondes to having fixed the real and the imaginary periods

$$
\begin{aligned}
& \omega=\int_{e_{3}}^{e_{2}} \frac{d t}{\sqrt{4 t^{3}-g_{2} t-g_{3}}}, \quad \omega^{\prime}=\int_{e_{2}}^{e_{1}} \frac{d t}{\sqrt{4 t^{3}-g_{2} t-g_{3}}}, \\
& \eta=\int_{e_{3}}^{e_{2}} \frac{t d t}{\sqrt{4 t^{3}-g_{2} t-g_{3}}}, \quad \eta^{\prime}=\int_{e_{2}}^{e_{1}} \frac{t d t}{\sqrt{4 t^{3}-g_{2} t-g_{3}}},
\end{aligned}
$$

as $2 \omega=1.82339,2 \omega^{\prime}=2.24176 i, 2 \eta=1.7851,2 \eta^{\prime}=-1.25119 i$ (note that Legendre's relation [5]: $\eta \omega^{\prime}-\eta^{\prime} \omega=i \frac{\pi}{2}$ is satisfied).

In real applications, since the $e_{i}$ 's determine the edges of the energy bands, one should consider them as parameters to be fixed by experiments. Let us start with the one gap potential ( $g=1$ in Eq. (27)) which, being simple, will be used to fix the notation and to check results. This potential is plotted in Fig. 1 as a function of $x, y$, from which we see that it is periodic (with the same period in both directions given by $\left.2\left|\omega^{\prime}\right|\right)$, real and well behaved over all space. In Fig. 2 the level curves of such potentials are reported in the Wigner-Seitz cell. From Eqs. (20) we have for this case only one equation $(g=k=1)$ which express the energy as a function of the "spectral" parameters: $E=\wp\left(a_{1}\right)+\wp\left(\tilde{a}_{1}\right)$. Since the energy is additive in the $\wp\left(a_{i}\right)$, the spectral parameters must vary on curves of the fundamental domain on which $\wp$ is real. These curves coincide with the intervals $[0,2 \omega]$ and $\left[\omega^{\prime}, 2 \omega+\omega^{\prime}\right]$ in correspondence of which the wavevectors $k_{x}=\zeta\left(a_{1}\right)-\frac{\eta^{\prime}}{\omega^{\prime}} a_{1}, k_{y}=\zeta\left(\tilde{a}_{1}\right)-\frac{\eta^{\prime}}{\omega^{\prime}}$ $\tilde{a}_{1}$ will span the BZ $\left[-\frac{\pi}{2\left|\omega^{\prime}\right|}, \frac{\pi}{2\left|\omega^{\prime}\right|}\right] \times\left[-\frac{\pi}{2\left|\omega^{\prime}\right|}, \frac{\pi}{2\left|\omega^{\prime}\right|}\right]$.

The energy bands in $k$-space are then obtained by parametrically plotting $E, k_{x}, k_{y}$ as functions of $a_{1}, \tilde{a}_{1}$ in the above intervals. This is shown in Fig. 3, from which we see that the ranges of the lower and upper bands respectively are $[-3,-1]$ and $[4, \infty]$. In terms of the spectral parameters this corresponds 
to taking $a_{1}, \tilde{a}_{1} \in\left[\omega^{\prime}, 2 \omega+\omega^{\prime}\right]$, for the lower band, and $a_{1}, \tilde{a}_{1} \in[0,2 \omega]$ for the upper one. This is evident from the fact that $\wp\left(\omega^{\prime}\right)=e_{3}=-1.5, \wp\left(\omega+\omega^{\prime}\right)=$ $e_{2}=-0.5$ and $\wp(\omega)=e_{1}=2$ (the bands edges are simply twice these values). Also note that for bands symmetric in $k$ (as in our case) one can restrict to only half of these parameter intervals (the other half just reproduce the symmetry). Since for a given band the energy is a continuous function of $k_{x}, k_{y}$, one can draw curves of constant energy analogous to the equipotential surfaces of electrostatics. One of these curves will be the so called Fermi curve, i.e. the curve for which all states described by $k$-vectors within it will be occupied and all $k$-vectors outside it will label unoccupied states. One can easily show that this curve (in the 3D case a surface) has the symmetry of the crystal, i.e. it is invariant under the point group operations characterizing the lattice.

In Fig. 4 we plot the Fermi curves, i.e. the curves of constant energy, as a function of $k_{x}, k_{y}$ for different values of $E$. Note that at the bottom and at the top of the bands, the Fermi curve are circles, as for the free electron case, whilst for intermediate values they deform and perpendicularly intersect the BZ boundaries. This fact is an indirect check on the correctness of our calculations since from the general theory it is known that the Fermi curve is always tangential to $\nabla E(k)$ and, for any reciprocal lattice vector $K$ perpendicularly joining opposite parallel faces of the BZ, one must have that $K \cdot \nabla E(k)=0$. In Fig. 5 the Bloch wave function at the bottom of the lower band (ground state) is shown. It is an extended function both in $x$ and $y$ and has maxima located in correspondence with the potential minima.

Let us now consider the case of two gaps in the spectrum $(g=2$ in Eq. (27)). From the system (20) we have two equations, one for the energy and another for $g_{2}$ as a function of the spectral parameters $a_{1}, a_{2}$. These equations can be easily solved by taking $a_{1}$ (similarly for $\tilde{a}_{1}$ ) varying on the 
same intervals as for the $g=1$ case (this is a requirement for the reality of the energy), and then solving the resulting quadratic equation for $\wp\left(a_{2}\right)$ involving $g_{2}$. By inverting the Weierstrass $\wp$ function we get $a_{1}, a_{2}$, which can then be used to plot the energy as a function of the wavenumbers. In Fig. 6 the two lower bands for the 2D two gap potential are plotted for the same parameters as before. In Fig. 7 we have shown the lowest band in higher detail (the highest energy band is similar to the one of $g=1$ and was omitted for graphical convenience). In Figs. 8, 9, a set of Fermi curves for the two lowest bands are also shown. From these figures we see for the lowest band the same qualitative behavior as for the $g=1$ case, but for the second band the Fermi curves are now asymmetrical with respect to the center of the Brioullin zone. In Fig. 10 the energy is plotted as a function of the spectral parameter along the lines $a_{i}=\tilde{a}_{i}$, from which we see that the edges of the bands are in agreement with the formula in Eq. (25) (in our case $g_{2}=13$ ).

A similar analysis can be done for the 3 gap case. Now besides the energy and the equation for $g_{2}$ we have also the equation for $g_{3}$. To find the spectral parameters $a_{1}, a_{2}, a_{3}$ in this case, one must numerically solve a quadratic and a sextic equation. Since the energy bands look qualitatively similar to the ones depicted in the previous cases, in Fig. 11 we show only the second lowest band and in Fig. 12 the corresponding Fermi curves. Note that the small gaps between the Fermi curves and the boundaries of the Brillouin zone are due to numerical effects (we stopped before intersecting the boundary since one needs higher precision i.e. longer computer time, to calculate the solution in these regions. It is evident, however, that the intersection of the Fermi curve with the edges of the Brillouin zone are always orthogonal. In Fig. 13 the energy bands are shown as functions of the spectral parameters. It is interesting to note that the shape of the bands are different from the ones in the reciprocal space (the second lowest band, for example, has a maximum in 
the center as a function of the spectral parameter, while it has a minimum as a function of the wavenumbers). The edges of these bands are

$$
E=0, \quad E=3 e_{i} \pm \sqrt{9 e_{j}^{2}+15\left(e_{j}-e_{k}\right)^{2}},
$$

plus cyclic permutations of $i, j, k=1,2,3$, which are just the roots of the polynomial in Eq. (26) (these values agree with the energy gaps derived by Smirnov in his reduction studies of elliptic potentials [21]).

Before closing this section we wish to discuss the case of lattices with rectangular Wigner-Seitz cells. This corresponds to the general case of different elliptic curves associated with each space direction. In Fig. 14 the level sets of a potential of this type are plotted (small circles correspond to maxima and large ones to minima). Here the periodicity in the $x$ direction is characterized by the same elliptic curve as before, but for the one in the $y$ direction we take the curve with parameters $\tilde{e}_{1}=4, \tilde{e}_{2}=-1, \tilde{e}_{3}=-3$ and with real and imaginary periods given by $2 \tilde{\omega}=1.82339,2 \tilde{\omega}^{\prime}=2.24176 i, 2 \tilde{\eta}=1.7851$, $2 \tilde{\eta}^{\prime}=-1.25119 i$. The Brillouin zone is then also rectangular with extensions $\left[-\frac{\pi}{2\left|\omega^{\prime}\right|}, \frac{\pi}{2\left|\omega^{\prime}\right|}\right] \times\left[-\frac{\pi}{2\left|\tilde{\omega}^{\prime}\right|}, \frac{\pi}{2\left|\tilde{\omega}^{\prime}\right|}\right]$. In Fig. 15 the two bands of the $g=1$ potential on a tectangular lattice are reported as function of wavevectors in the first BZ. In contrast with the square lattice case, both bands are asymmetric in the two directions, with edges given by $-4.5,-1.5,6$. These numbers are just the sums of the edges of the two separate elliptic curves, $e_{1}+\tilde{e}_{1}, e_{2}+\tilde{e}_{2}$, $e_{3}+\tilde{e}_{3}$. Moreover, from Fig. 16 we see that, due to the asymmetry, the Fermi curves intersect the Brioullin zone boundaries first in $k_{y}$ and then in the $k_{x}$ direction.

It is interesting to note that, although the Fermi curve approaches the boundary tangentially, after the intersection it always becomes orthogonal to it. We also note that at the bottom and at the top the behavior is very similar as for the square lattices, i.e. the curves are circles as for free electrons. From 
this analysis one can easily get a qualitative picture of the behavior of the bands and of the Fermi curves for the multigap 2D potentials on rectangular lattices, and therefore we will not discuss them further here. In closing this paper we wish to remark that with semiconductor nano-technologies, it is possible today to construct artificial solids in 2D dimensions as arrays of quantum dots [22]. With electrode deposition techniques [23], it could indeed be possible to construct periodic gates on top of a quantum well so as to create smooth $2 \mathrm{D}$ potentials of the type described in this paper. Moreover, the generalization of our analysis to 3D potentials, with a Wigner Seitz cell which is a cube or a rectangular parallelogram, can easily be done.

We hope to generalize our results to lattices of some other spatial symmetry by means of the Trebich-Verdier and Smirnov potentials (see e.g. [24]). But the extension of this method to lattices of general spatial symmetry with separable multidimensional Lamé potentials seems problematical. In this case, however, potentials arising from hyperelliptic extensions of this theory, can be useful. This generalization is presently under investigation [25].

Acknowledgments The research of the second and fourth authors was supported in part by the INTAS grant 93-1324-Ext. The research of the third author was supported in part by the CRDF grant and INTAS grants 93-1324Ext and INTAS-96-770. JCE and MS are also grateful for support under the LOCNET EU network HPRN-CT-1999-00163. 


\section{REFERENCES}

[1] F. Bloch. Quantum mechanics of electrons in crystal lattices. Zeits. f. Physik, 52:555-600, 1928.

[2] G. H. Halphen. Traité des Fonction Elliptiques, volume 2. Gauthier-Villars, Paris, 1888.

[3] M. Krause. Theorie der doppeltperiodischen Funktionen einer veränderlichen Grösse, volume 1. Teubner, 1895.

[4] A. R. Forsyth. Theory of Differential Equations. Dover, 1959. Part III, Vol. 4.

[5] E. T. Whittaker and G. N. Watson. A Course of Modern Analysis. Cambridge University Press, 1969.

[6] F. M. Arscott and I. M. Khabaza. Tables of Lamé Polynomials, volume 17 of Mathematical tables series. Pergamon, Oxford, 1962.

[7] F. M. Arscott. Periodic differential equations: an introduction to Mathieu, Lamé, and allied functions, volume 66 of International series of monographs in pure and applied mathematics. Pergamon, Oxford, 1964.

[8] I. M. Krichever. Elliptic solutions of the Kadomtsev-Petviashvili equation and integrable systems of particles on a line. Funct. Anal. Appl., 14:282-290, 1980.

[9] J. C. Eilbeck and V. Z. Enol'skii. Elliptic Bake-Akhiezer functions and an application to an integrable dynamical system. J. Math. Phys., 35:1192-1201, 1994.

[10] V. Z. Enol'skii and N. A. Kostov. On the geometry of elliptic solitons. Acta Appl.Math., $36: 57-58,1994$.

[11] A. O. Smirnov. Finite-gap elliptic solutions of the KdV equation. Acta Appl. Math., 36:125$166,1994$.

[12] F. Gesztesy and R. Weikard. Lamé potentials and the (m)KdV hierarchy. Math. Nachr., 176:73-91, 1995. 
[13] F. Gesztesy and R. Weikard. Elliptic algebro-geometric solutions of the KdV and AKNS hierarchies - an analytic approach. Bull. AMS, 35:271-317, 1998.

[14] A. Zabrodin. On the spectral curve of the difference Lamé operator. IMRN, 11:589-614, 1999.

[15] V. G. Bar'yakhtar, E. D. Belokolos, and A. M. Korostil. A new method for calculating the electron spectrum in solids: application to high-temperature superconductors. Phys. Stat. Sol.(b), 169:105-114, 1992.

[16] V. G. Bar'yakhtar, E. D. Belokolos, and A. M. Korostil. Method of separable finite-band potential: a new method for calculating electron energy spectrum of solids. Phys. Metals, 12:829-838, 1993.

[17] A. Krazer. Lehrbuch der Thetafunctionen. Teubner, 1903. reprinted by Chelsea, 1998.

[18] E. D. Belokolos, A. I. Bobenko, V. Z. Enol'skii, A. R. Its, and V. B. Matveev. Algebro Geometrical Aproach to Nonlinear Integrable Equations. Springer, Berlin, 1994.

[19] W. A. Harrison. Pseudopotentials in the Theory of Metals. Benjamin, New York, 1966.

[20] G. Floquet. Sur les equations différentielles linéaires á coefficients périodiques. Ann. Sci. École Norm., Super. (2), XII:47-89, 1883.

[21] A. O. Smirnov. On a class of elliptic potentials of the Dirac operator. Math USSR Sbornik, 188:115-135, 1997.

[22] L. Jacak, P. Hawrylak, and A. Wojs. Quantum Dots. Springer, 1998.

[23] J. P. Kotthaus J. Alsmeier, E. Batke. Voltage-tunable quantum dots on silicon. Phys. Rev. B, 41:1699-1702, 1990.

[24] V. G. Bar'yakhtar, E. D. Belokolos, and A. M. Korostil. Fermi-surfaces of metals with $f c c$ structures in the model of finite-band potential. Metalofizika, 15:3-13, 1993. (in Russian).

[25] V. M. Buchstaber, V. Z. Enol'skii, J. C. Eilbeck, D. V. Leykin, and M. Salerno. 2D Schrödinger 
equations with Abelian potentials. In preparation, 2000. 


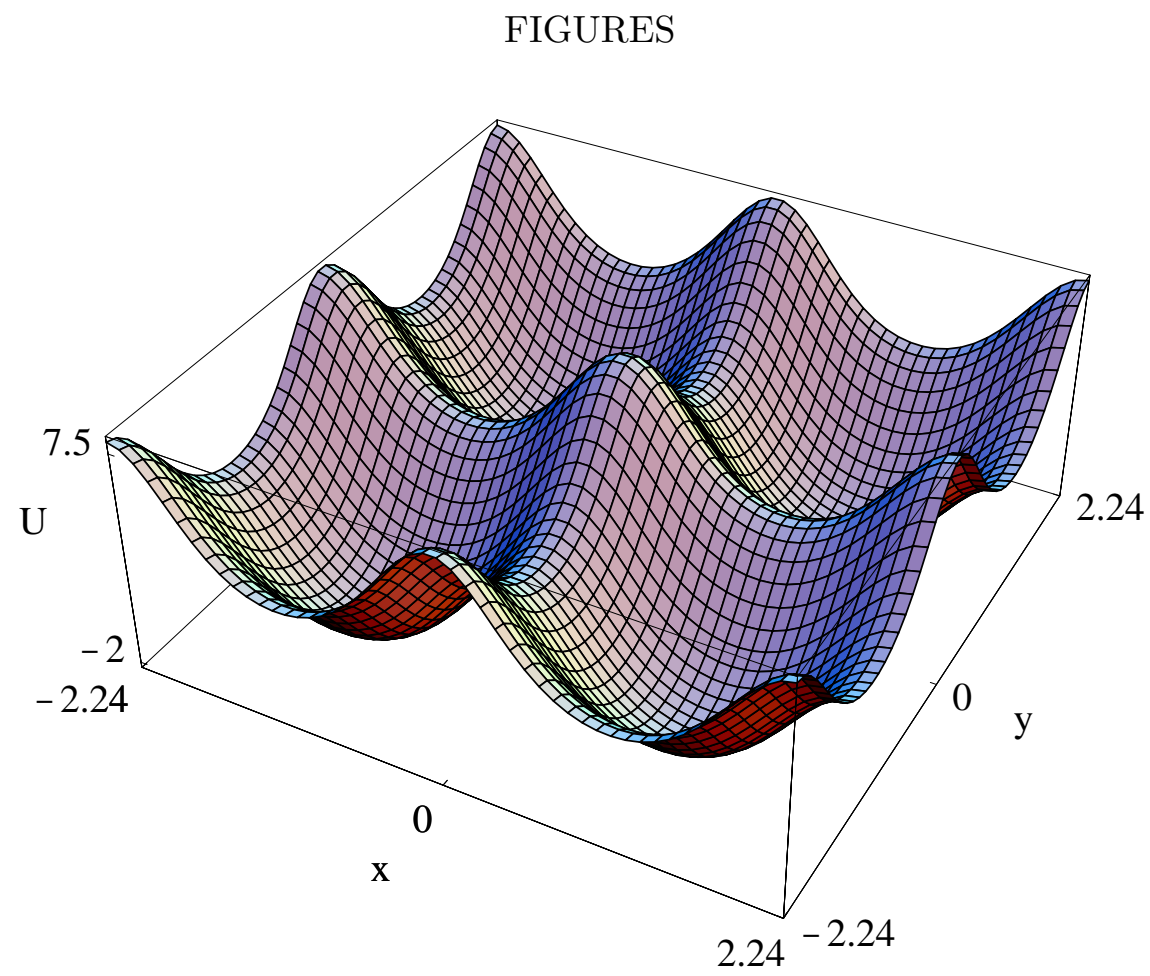

FIG. 1. Potential profile in the one-gap case with parameters values $e_{1}=2, e_{2}=-0.5, e_{3}=-1.5$

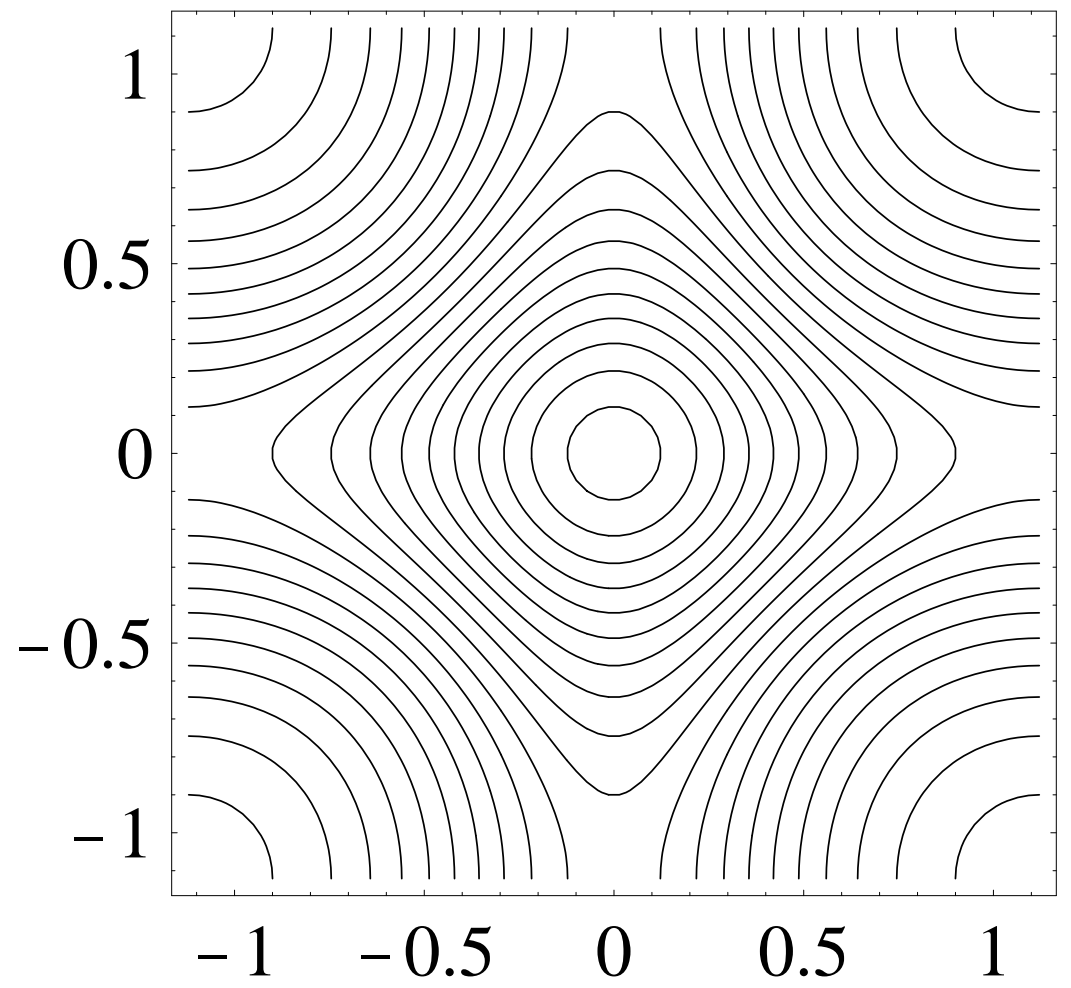

FIG. 2. Level curves of the potential in Fig. 1 


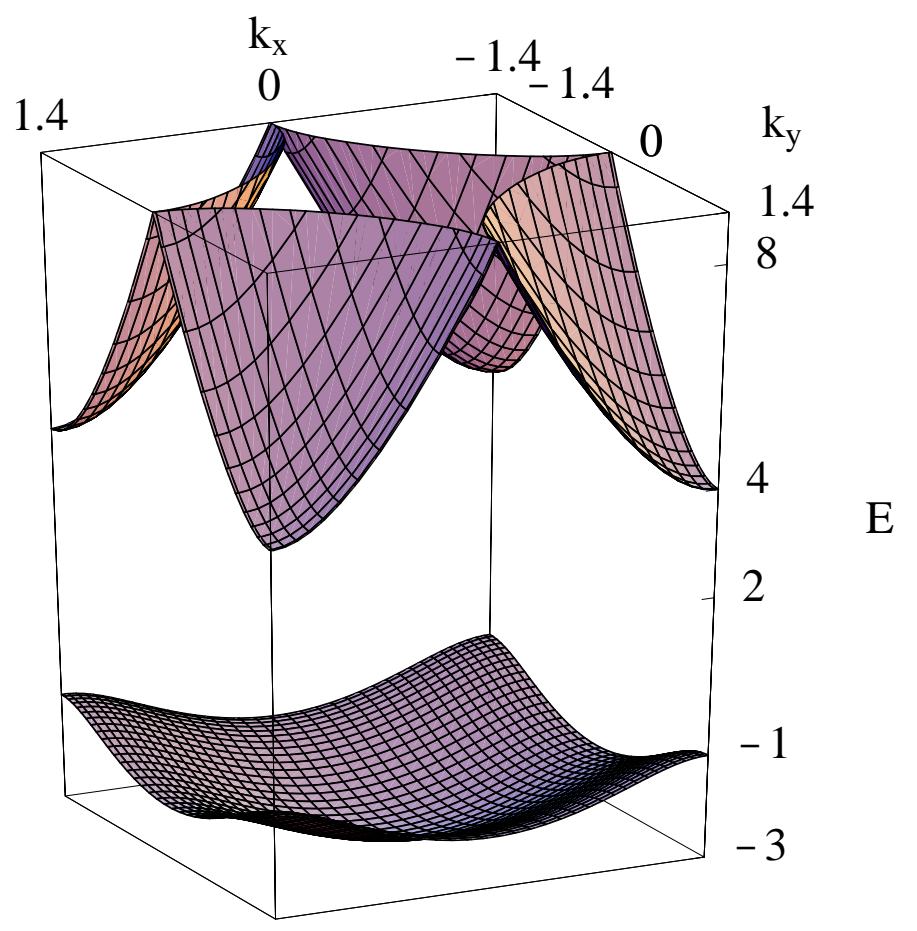

FIG. 3. Energy bands of the first Brillouin zone for the potential in Fig. 1. The edges of the Brillouin zone (i.e. $\pm \frac{\pi}{2\left|\omega^{\prime}\right|}$ ) for the parameters chosen in Fig.1 correspond to the values \pm 1.401396
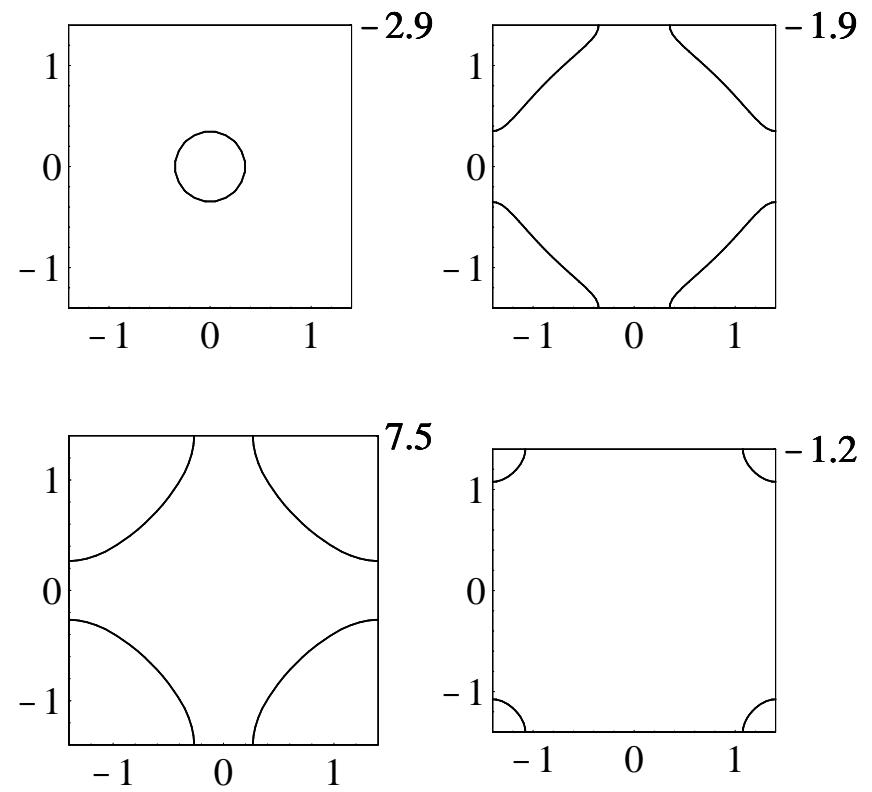

FIG. 4. Fermi curves relative to the energy bands of Fig. 3. The constant energy value at which the curves are taken is reported at the right top of each figure 


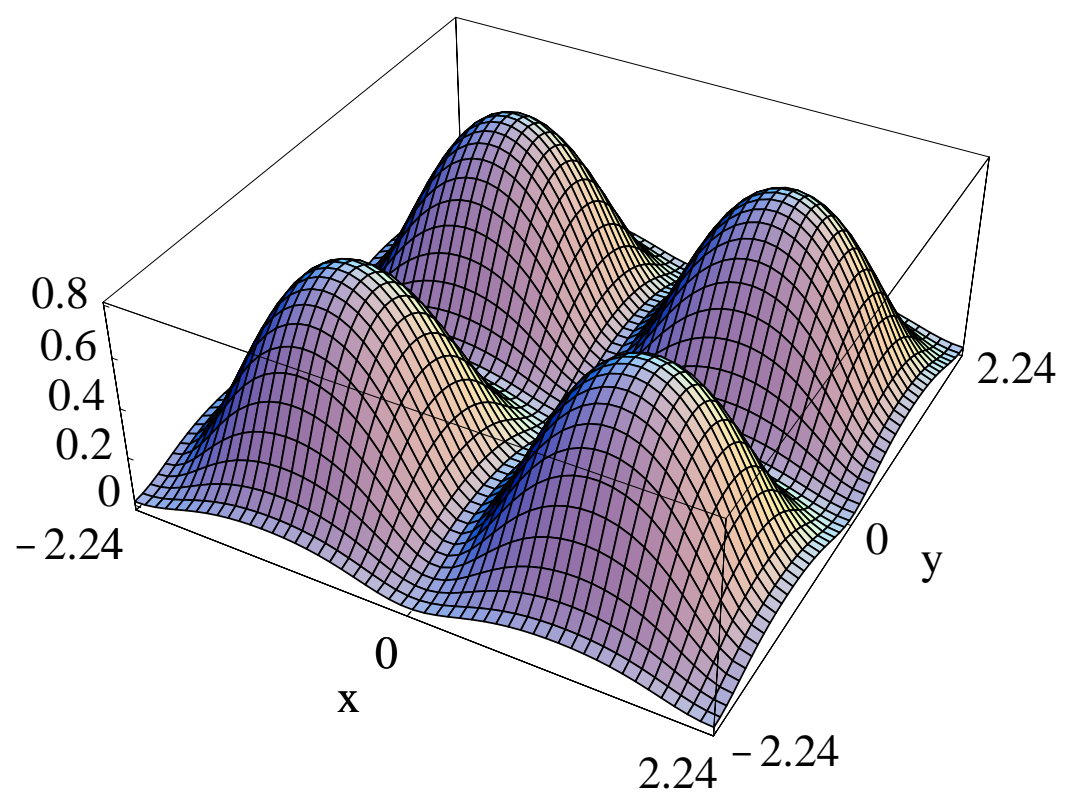

FIG. 5. Modulo squared of the wave function of the ground state for the potential in Fig. 1. The energy is $E=-3$ corresponding to the bottom of the first band in Fig. 2. 


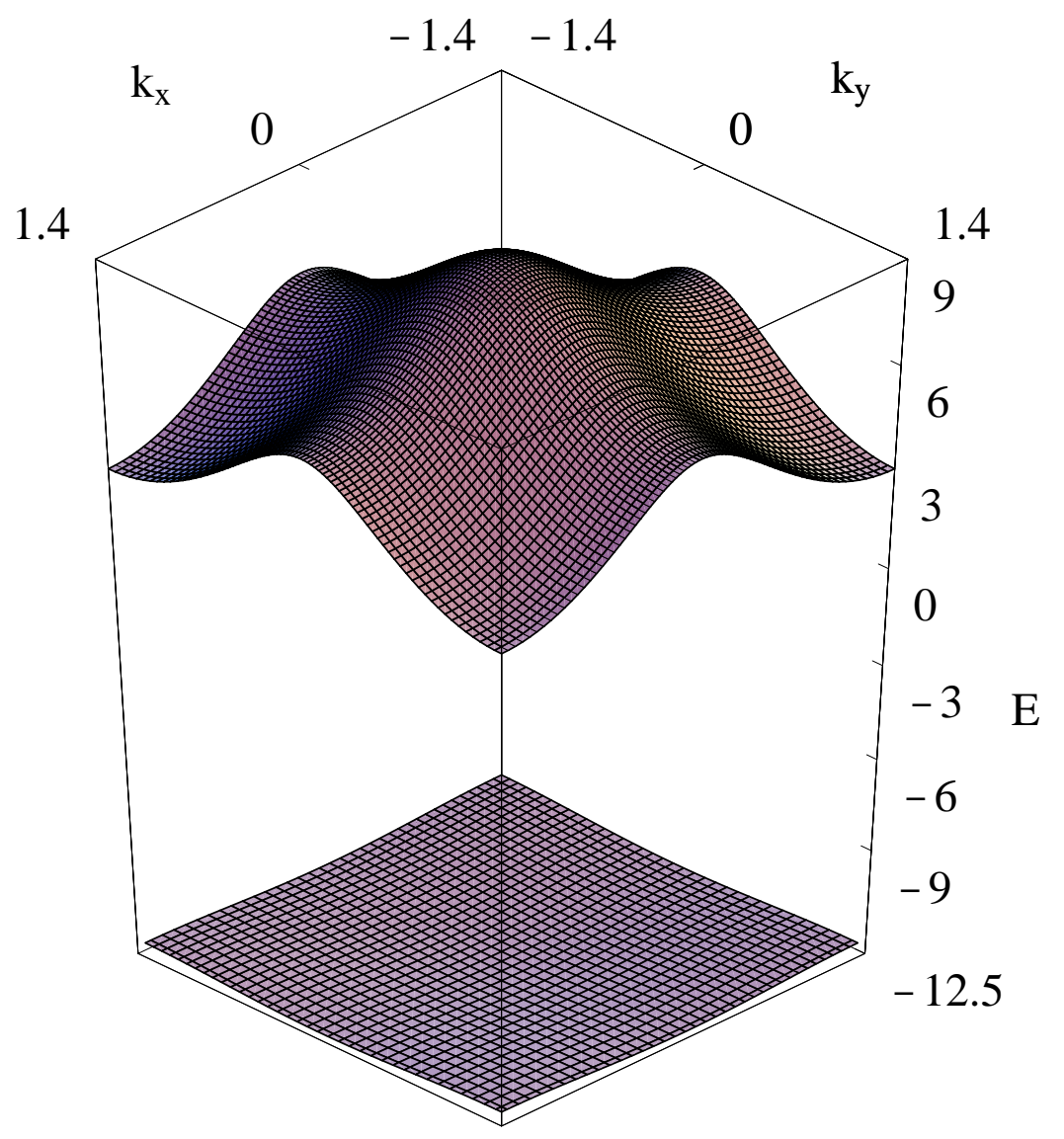

FIG. 6. The lowest two energy bands for separable two gap potential characterized by the parameters given in Fig. 1

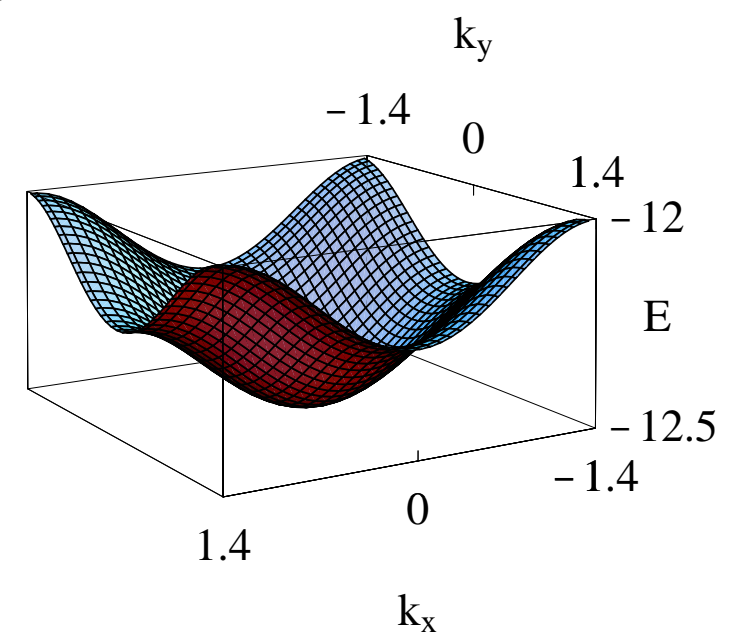

FIG. 7. The lower band in Fig. 6 reported in a larger scale 

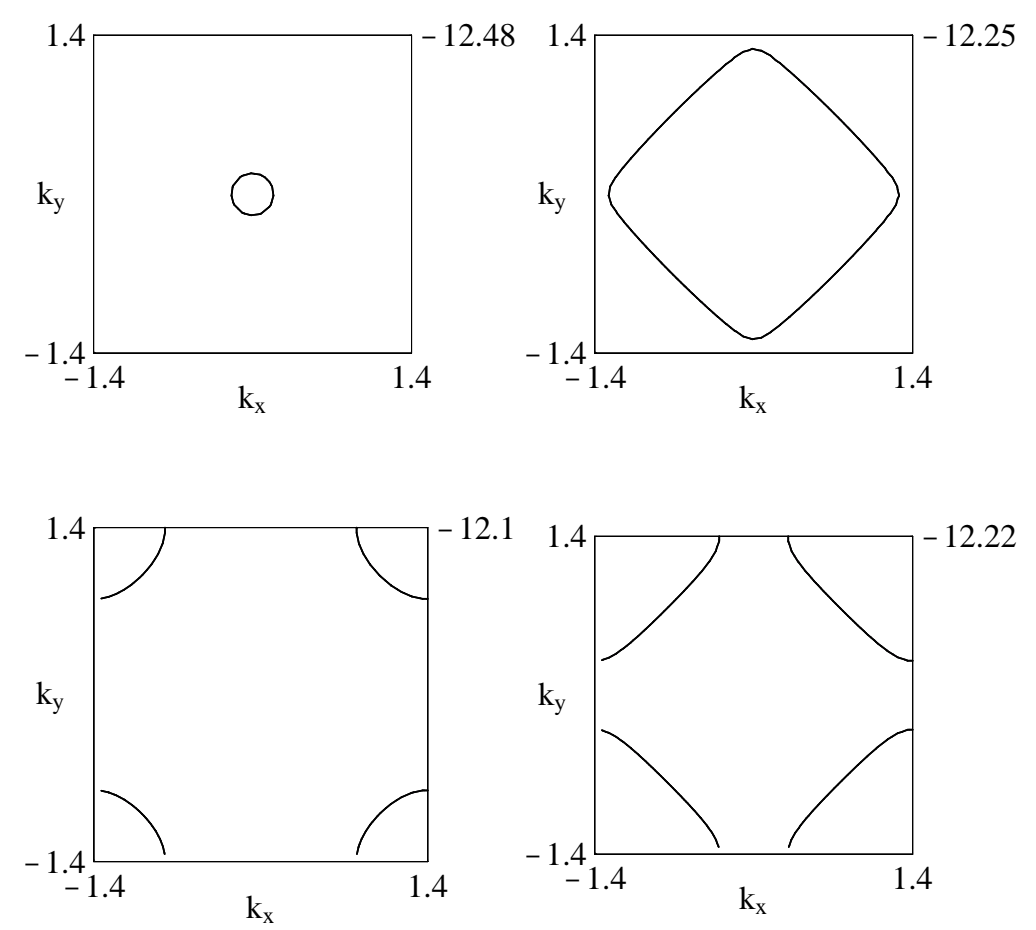

FIG. 8. Fermi curves relative to the first (lower) band of Fig. 6. The top right of each figure shows the constant energy value at which the curve is taken.
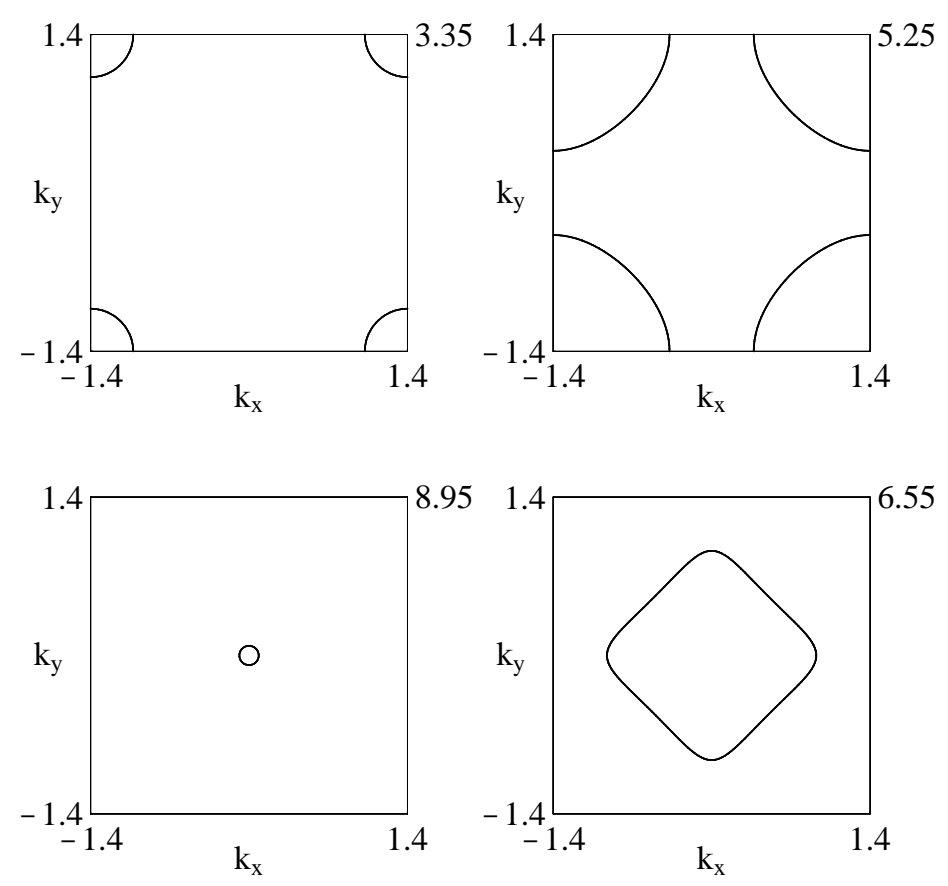

FIG. 9. Same as in Fig. 8 but for the second band of Fig. 6 . 


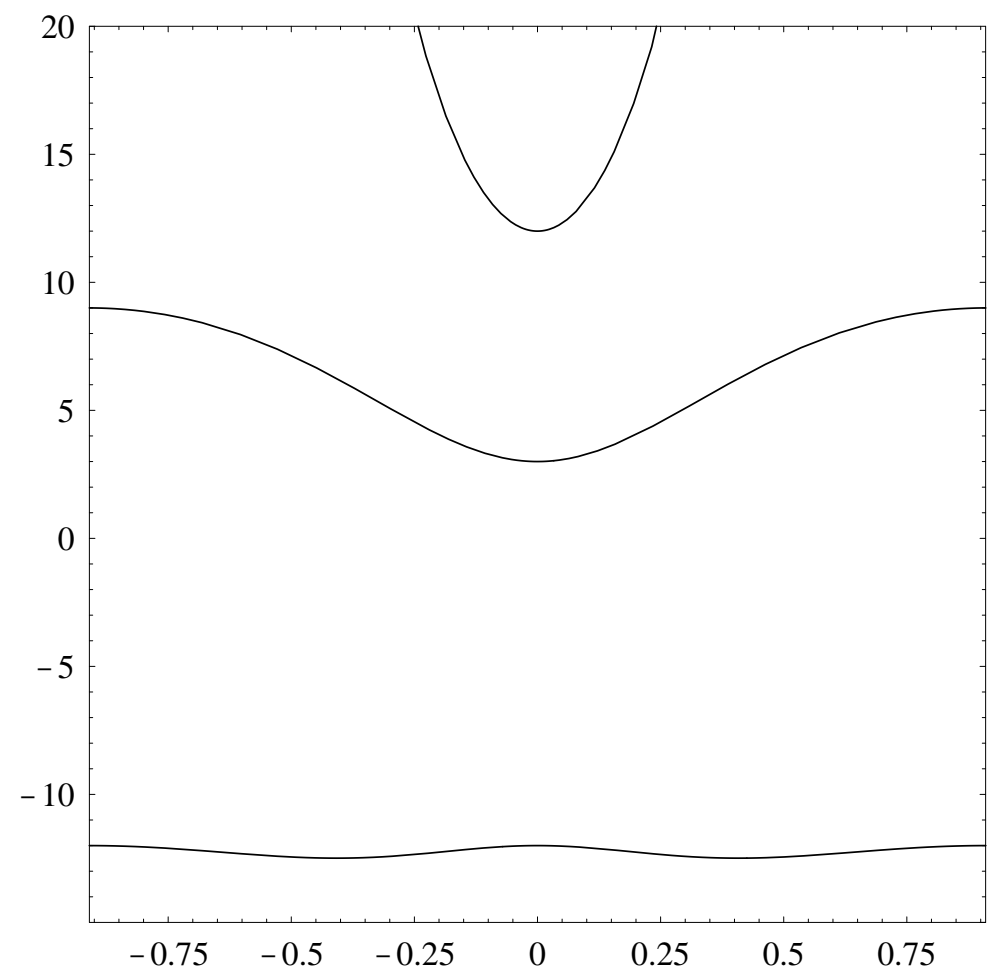

FIG. 10. Energy curves as functions of the spectral parameters for $\alpha=\beta \in\left[-\omega / 2, \omega^{\prime} / 2\right]$. The edges of the energy bands are $-2 \sqrt{3 g_{2}},-6 e_{1},-6 e_{2},-6 e_{3}, 2 \sqrt{3 g_{2}}$, with $e_{1}, e_{2}, e_{3}$ as in Fig. 1

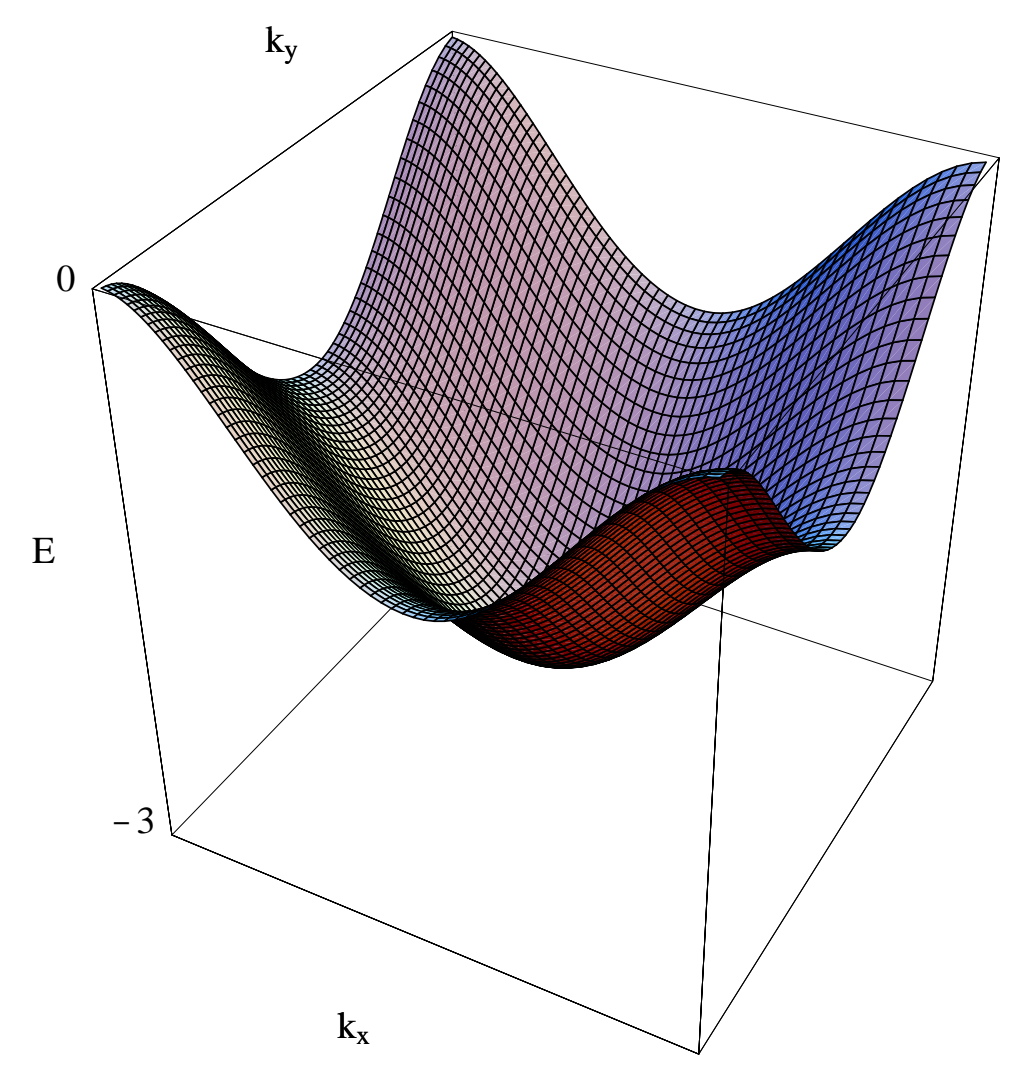


FIG. 11. Second lowest energy band for the 3-gap case. The parameters of the curves are fixed as in Fig. 1

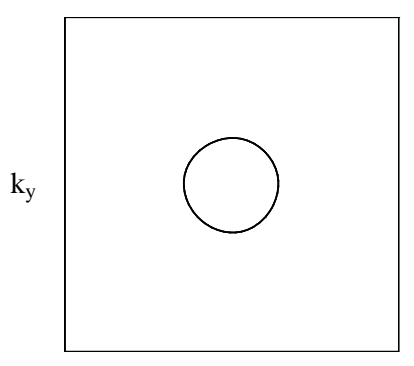

$\mathrm{k}_{\mathrm{x}}$

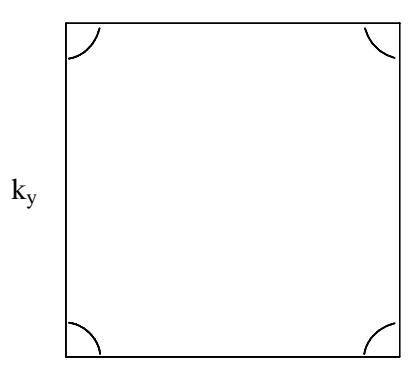

$\mathrm{k}_{\mathrm{x}}$
$-2.1$
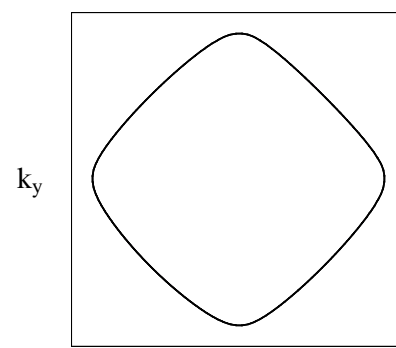

$\mathrm{k}_{\mathrm{x}}$

$-0.15$

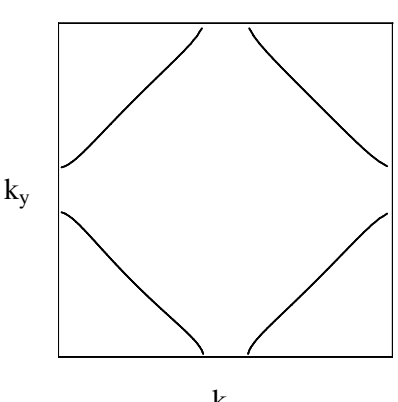

$-1.2$

$-1.1$

FIG. 12. Fermi curves relative to the band in Fig. 11.

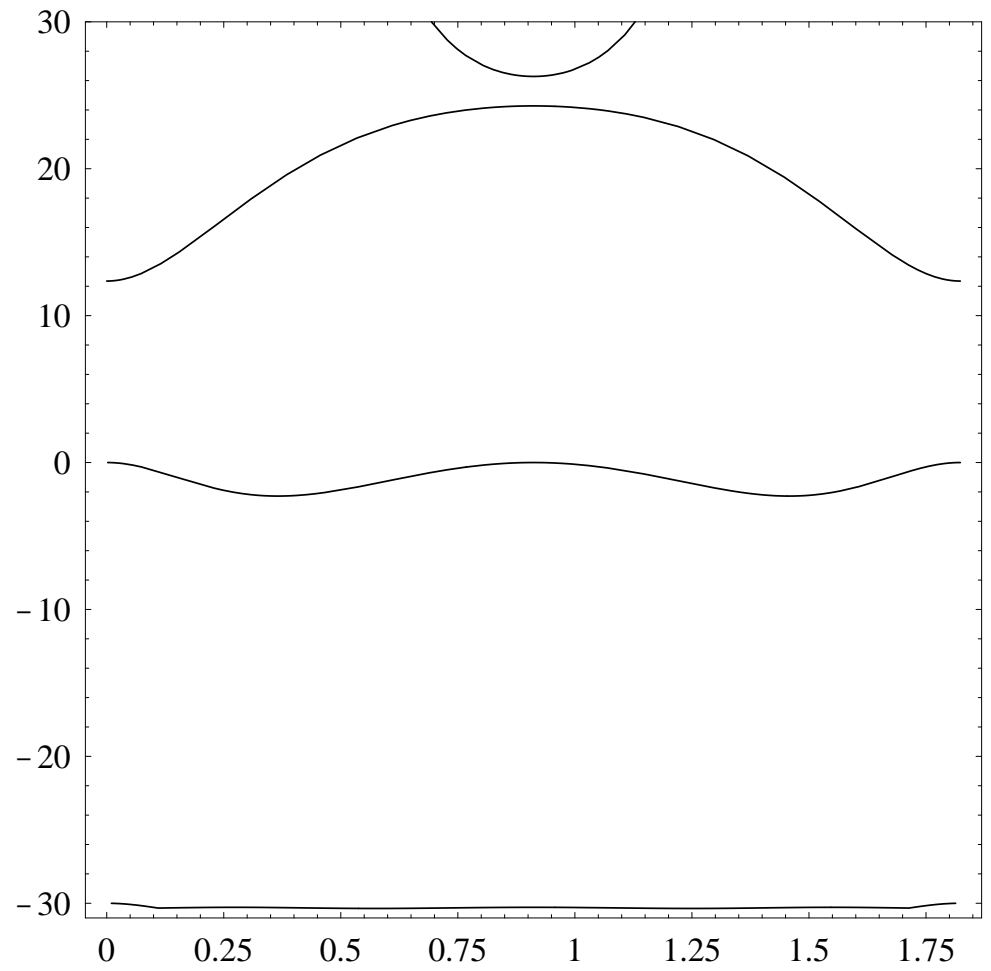

FIG. 13. Energy curves as functions of the spectral parameters for $\alpha=\beta \in[0,2 \omega]$ for the 3-gap case. The edges of the energy bands are the root of the polynomial in Eq. (26). 


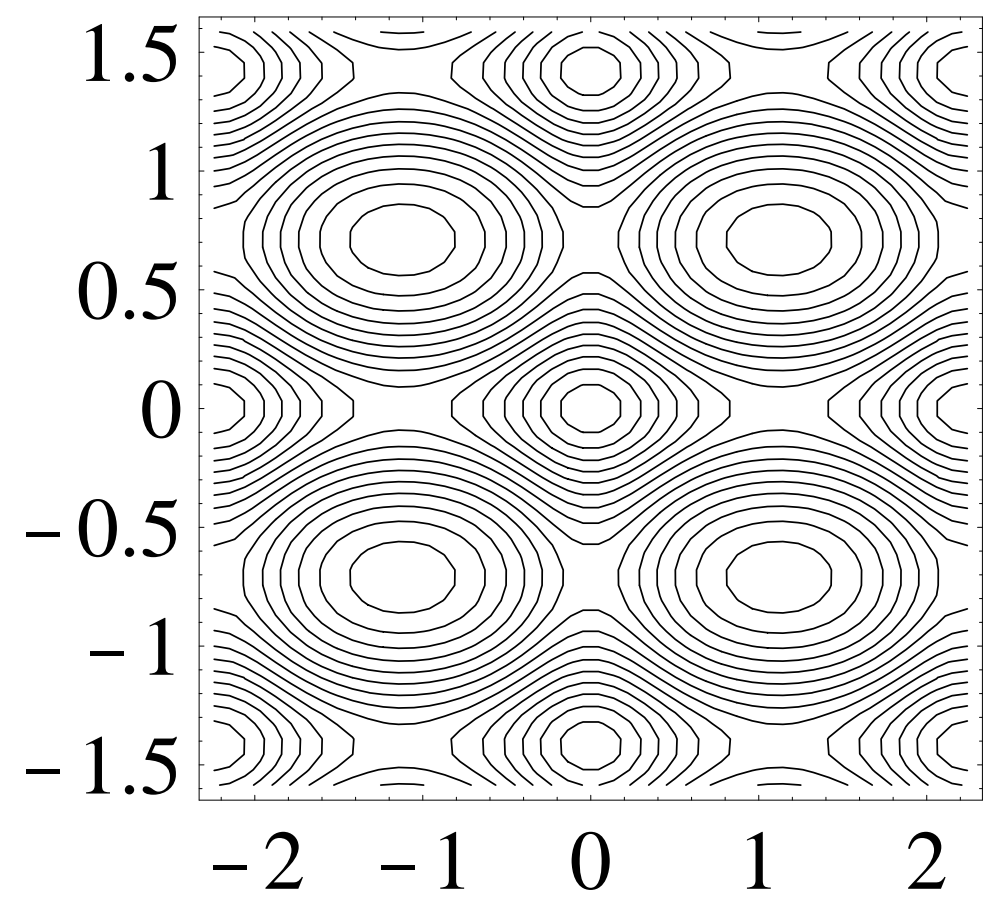

FIG. 14. Level curves of 1-gap potential characterized by two different curves. The first curve characterizing the $x$ direction has the same parameters as in Fig. 1, whilst the other has parameter $\tilde{e}_{1}=4, \tilde{e}_{2}=-1, \tilde{e}_{3}=-3$. 


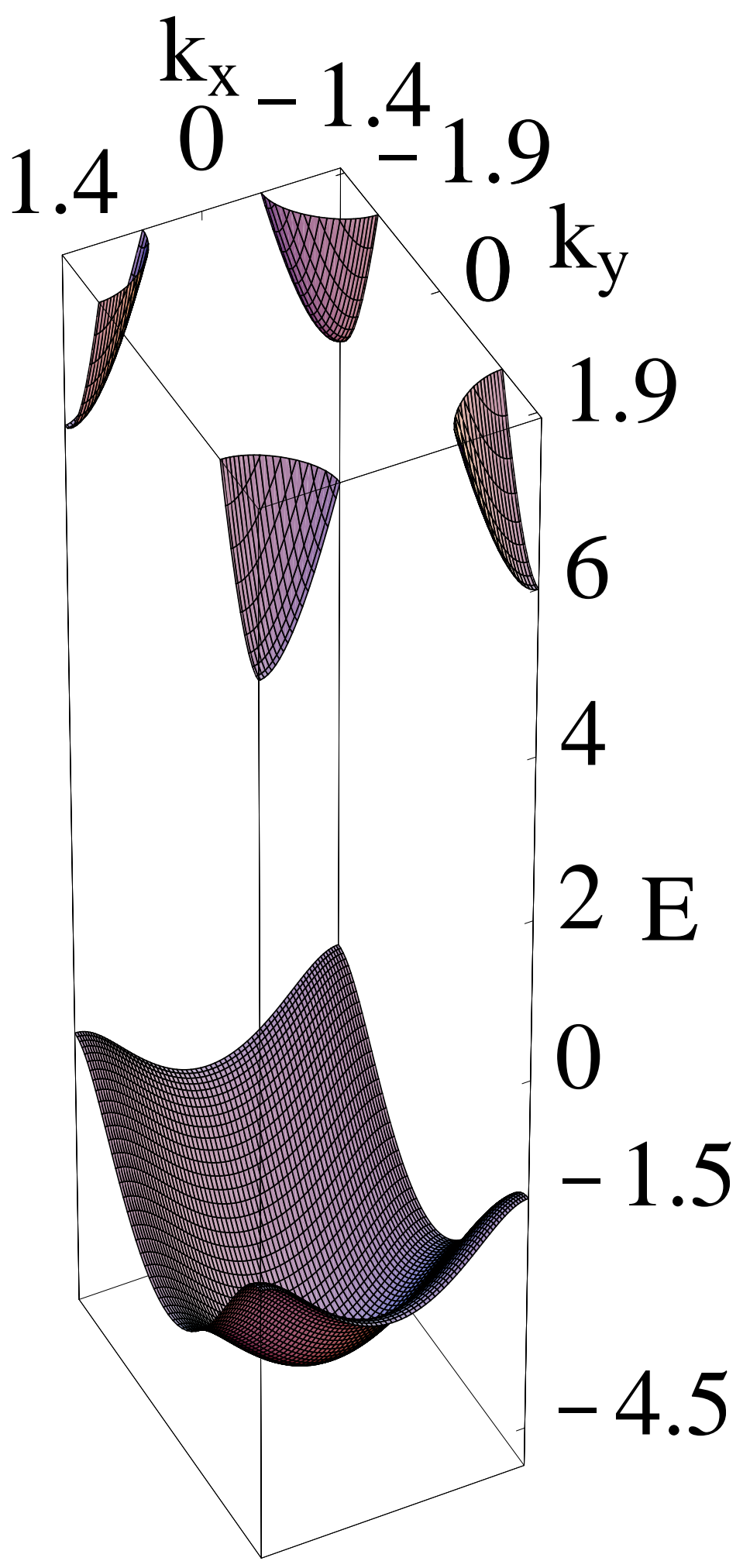

FIG. 15. Energy bands in the first Brillouin zone for the potential in Fig. 14. 

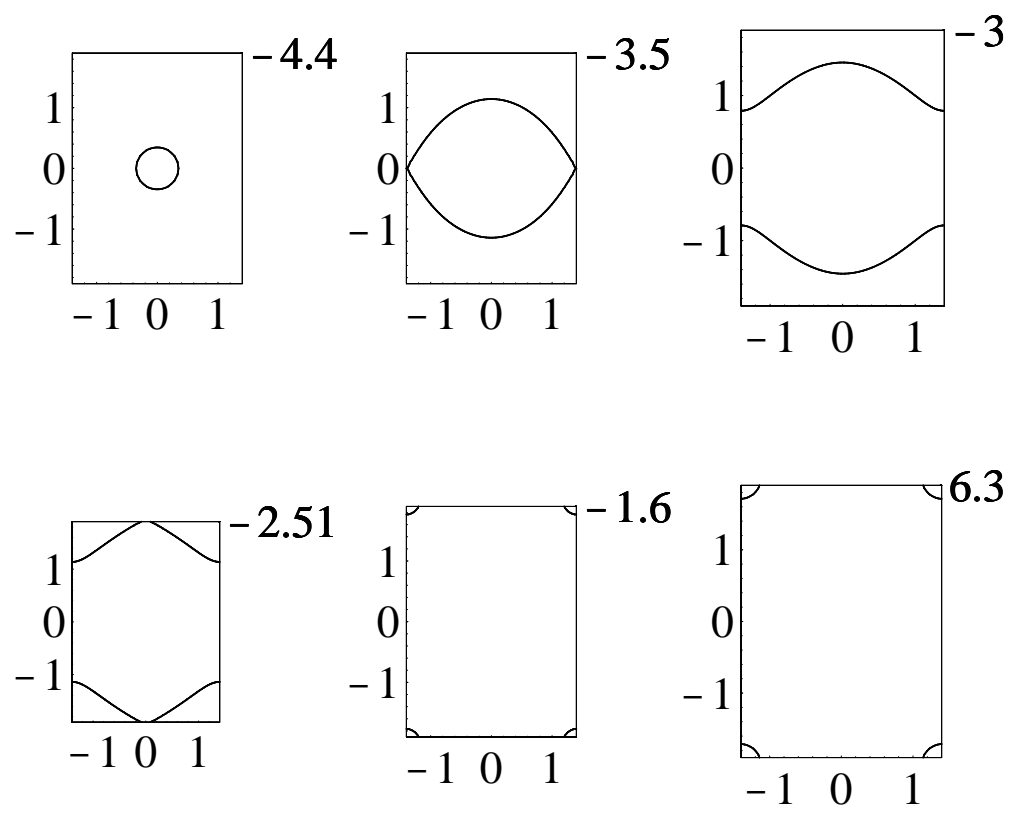

FIG. 16. Fermi curves in the first Brillouin zone for the bands in Fig. 15. 